\title{
Ergebnisse der Untersuchung von Ersatzmitteln.
}

\author{
Januar-März 1917. \\ Von
}

Dr. K. Krafft,

Vorstand des Chemischen Laboratoriums des K. Württembergischen Medizinalkollegiums in Stuttgart.

[Eingegangen am 12. April 1917.]

Im Anschluß an die frühere Mitteilung ${ }^{1}$ ) folgt eine solche über weitere Ergebnisse der im Chemischen Laboratorium des K. Württembergischen Medizinalkollegiums ausgeführten Untersuchungen von Ersatzmitteln.

\section{a) Fleischersatzmittel u. dergl.}

„Milfix Kunst-Fleisch“ (29. I. 17), „Vollkommener Fleischersatz in Nährwert, Geschmack, Aussehen, Anwendung und chemischer Zusammensetzung"“. Der Inhalt einer Dose entspreche nach Angabe in Gewicht und Eiweißgehalt annähernd dem aus einem Pfund frischen Kalbfleisches erhaltenen Kochfleisch usw. Inhalt einer Büchse 300 g. „Preis 2 Mk. 20 Pfg. Hergestellt Oktober 1916. Milfix G. m. b. H. Berlin S. O. 29". Eine gelatinöse, dünne Schicht und einige große, weißgelbliche bis bräunliche Stücke, an denen dunklere, schwammige Teile saßen. Nach kurzem Lagern im warmen Raume trennte sich eine dunkelbraune Brühe mit Hefegeruch $a b$ und die Stücke umgaben sich mit einer harten Schicht, die widerlich leimartig roch. Beim Kochen in Wasser nahmen die zerkleinerten Stückchen eine härtere, fast lederartige Beschaffenheit an und wurden fast weiß. Beĭ näherer Betrachtung war festzustellen, daß hier ein Röhrenpilz vorlag, an dessen Hute noch Teile des schwamnigen, dunkleren Hymeniums hafteten.

\section{Chemischer Befund:}

\begin{tabular}{|c|c|c|c|c|c|}
\hline Wasser . . & . & . & $\cdot \cdot \cdot$ & $\cdot \cdot$ & $70,69 \%$ \\
\hline Stickstoffsubstanz & $\mathrm{z}$. & . & . . & & 23,60 \\
\hline Fett . . . . & . & . & . & . & 0,35, \\
\hline Asche . . & . & . & . & . & $3,73 \%$ \\
\hline Chlornatrium . & & . & . & . & $1,90 \%$ \\
\hline Phosphorsäure (P & $\left.\mathrm{P}_{2} \mathrm{O}_{5}\right)$ & " & . & . & $1,22 "$ \\
\hline Qualitativ . . & & . & . & & Gelatine nachgewiesen. \\
\hline Nährwert für & r $1 \mathrm{~kg}$ & & a) nach & König & $\begin{array}{l}\text { b) nach der Kriegs- } \\
\text { sanitätsordnung }\end{array}$ \\
\hline Fett. & & & 32,35 & Kalorien & 31,5 Kalorien \\
\hline tickstoffsubstanz & & & 1328,37 & 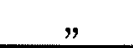 & $802,74 \quad$ \\
\hline
\end{tabular}

Sonach bestand das Milfix-Kunst-Fleisch aus Teilen eines Röbrenpilzes, die zur Erhöhung der Stickstoffsubstanz mit einer Mischung von Gelatine und Nährhefe übergossen waren.

"Viandal Deutseher Kraft-Extrakt" (15. II. 17). Angegebenes Datum der Herstellung: 28. November 1916. „Viandalgesellschaft deutscher Kraftprodukte

1) Diese Zeitschrift 1917, 88, 369. 
m. b. H. Berlin W 35. Netto $75 \mathrm{~g}=1 \mathrm{Mk}$. 45 Pfg." In Porzellantopf gefülltes dunkelbraunes, zähes, körniges Extrakt mit Geruch nach Fleischextrakt und stark salzigem Geschmack.

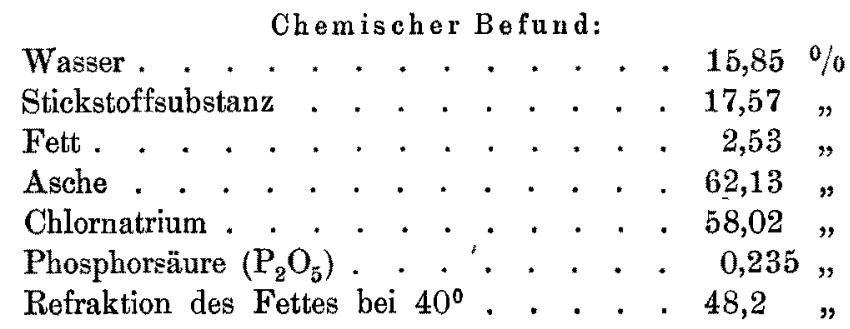

Hiernach konnten $75 \mathrm{~g}$ dieses Erzeugnisses (unter Abzug von $58 \%$ Kochsalz und $16 \%$ Wasser) nur rund $20 \mathrm{~g}$ Fleischextrakt enthalten (=1 Mk. 45 Pfg.!).

\section{b) Eier-Sparmittel, Backpulver u. dergl.}

„Eier-Sparpulver Eiola (Eigelbfarbe mit Triebkraft); Färbegehalt von 5-6 Hühnereiern. Hergestellt aus Lockerungsstoffen und Farbzusatz" usw. (14. II. 17) Fabrik chemischer Produkte A dolf Schähfer in Ludwigshafen a. Rh., $16 \mathrm{~g}$ $=15$ Pfg., bestand aus einem Gemenge von kohlensaurem Kalk $(66,4 \%)$ und saurem schwefligsaurem Natrium, das durch Eisen, Silicium und Magnesium verunreinigt war, sowie Kochsalz unter Beimischung eines künstlichen Farbstoffs.

"Germania Backpulver, Hoflieferant Th. Franz \& Co. Halle a. S." (31. I. 17) $250 \mathrm{~g}=1 \mathrm{Mk}$. $25 \mathrm{Pfg}$., bestand zur Hauptsache aus einem Gemisch von saurem Calciumphosphat (55\%), doppeltkohlensaurem Natrium $(29 \%)$ und geringen Mengen saurem weinsaurem Kalium, das als Verunreinigung Gips und Eisenphosphat enthielt.

\section{c) Nährmittel u. dergl.}

„Hygiomat" (29. I. 17) 1 Pfund $=2 \mathrm{Mk} .75 \mathrm{Pfg}$., war nach der chemischen Untersuchung ein durch Diastasierung und mehrfachen Backprozeß erhaltenes Gerstenmehlerzeugnis, welchem Zucker zugesetzt war:

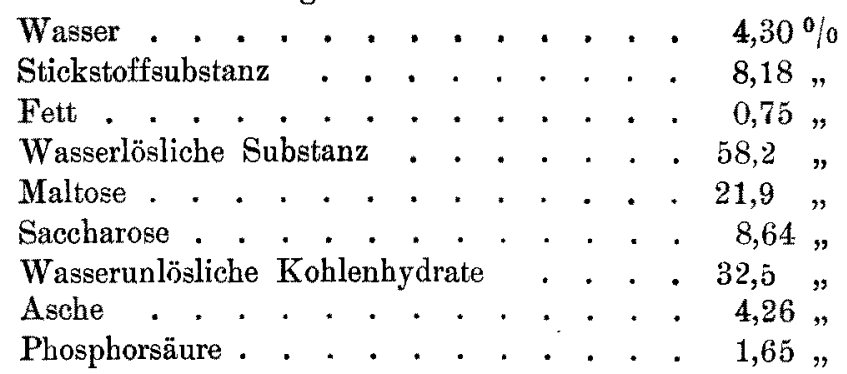

„Ilnur, Nährspeisemehl ohne Aroma. William Lederhausen, Deutsche Ifnurwerke, Hamburg 22" (14. II. 17). $225 \mathrm{~g}=1 \mathrm{Mk}$. 30 Pfg., bestand aus einem Gemenge voń Gersten- und Maismehl; kleine Mengen Roggenmehl und Unkrautsamen waren wohl nur zufällige Verunreinigungen. 


\section{d) Puddingpulver u. dergl.}

„Erkah-Creme, ges. gesch. Zitronen-Geschmack, leicht gefärbt. Roth \& Co. Chemische Fabrik, Berlin S 14, August 1916" (1. III. 17), $40 \mathrm{~g}=45$ Pfg., war eine Mischung von ungefähr je gleichen Mengen von Kartoffel-, Mais-, Reis- und Gerstenmehl, welcher etwas gepulverte Citronenschalen und Farbstoff zugesetzt worden waren.

„Dr. Strassmann's Pudding.Pulver mit Mandeln und Rosinen. Dr. Strassmann Akt. -Ges. Chem. und Nährmittelfabrik Berlin, Charlottenstr. 95. Inhalt ca. $40 \mathrm{~g}$, Oktober 1916, Preis 40 Pfg., leicht gefärbt" (1. III. 17) bestand aus einer Mischung von ungefähr gleichen Mengen Mais- und Gerstenmehl, sowie weniger Kartoffelstärke in verkleistertem Zustand und kleineren Mengen (fettarmer) Mandeln. Auferdem waren dem Gemenge Rosinen und etwas Vanillin und Farbstoff zugesetzt.

\section{e) ,Käse-Geschmack $๘$.}

„Liptauer Käse-Geschmack Marke Tischlein deck dich. Pharmazeut. Fabrik A. Klein G. m. b. H. Berlin SW, Kochstr. 57" (7. II. 17). Verpackung: Bunte, der Gestalt von Käsepackungen gleiche Faltschachtel mit vorerwähntem Aufdruck. Inhalt: $10 \mathrm{~g}$ grobes Pulver. Preis $25 \mathrm{Pfg}$. Geruch nach Baldriansäureester. Geschmack stark scharf und salzig. Das vorgelegene Erzeugnis bestand aus Kochsalz (96\%), dem geringe Mengen Paprika und Kümmel beigegeben waren, und das zur Vortäuschung des Käsegeruches mit Baldrianester übergossen war.

\section{f) Suppen-Mischungen.}

„Gemüsesuppe Marke Agnes, leicht gefärbt. Hergestellt November 1916. Inhalt $20 \mathrm{~g}$ für 4 Teller Suppe. Nährmittelfabrik J. Cronheim, Hamburg, Bremfelderstr. 84" (20. II. 17), Preis 15 Pfg. Das Erzeugnis war ein Gemenge von Kochsalz $(79 \%)$, Nährhefe und getrockneten Kräutern mit künstlicher Färbung.

„Omaco-Familien-Suppe, Omaco-Werk Coswig i. Sa. Kötschenbroda, Fernsprecher Nr. 7, handelsüblich gefärbt" (26. II. 17), $25 \mathrm{~g}=20 \mathrm{Pfg}$., war ein Gemenge von Kochsalz $(17 \%)$, Nährhefe und gequetschten Gerstenkörnern, das Spuren Suppenkräuter (Karotten- und Gemüseteilchen) und als Verunreinigung etwas Unkräuter, wie Wicke und Kornrade, enthielt.

„Omaco-Gemüse-Suppe, Omaco-Werk Coswig i. Sa., Fernsprecher-Amt Kötschenbroda Nr. 7“ (26. II. 17), $25 \mathrm{~g}=20$ Pfg., war ein Gemenge von gepulverter Bohne, Erbse, Gerste und getrockneter Kartoffel und Suppenkräutern (u. a. Karotten und Lauch) unter Zusaiz von etwas Nährhefe und 20\% Kochsalz.

\section{g) Gelee- und Marmelade-Ersatz.}

„4 Früch te-Gelee-Pulver, bester Ersatz für Nährfrüchte zur Selbstbereitung einer vorzüglichen Gelee-Marmelade" (5. II. 17), Hersteller nicht genannt, in Papierbeutel verpackt. $75 \mathrm{~g}=45$ Pfg. Das körnige, grobe, rotgelbe Pulver, welches nach Estern roch und süß und zugleich sauer schmeckte, war ein Gemenge von Zucker (69\% Saccharose), Gelatine und Citronensäure $(4 \%)$, das mit einem Teerfarbștoff rötlich gefärbt war.

"Marmelade". Ein unter diesem Namen unter Nachnahme Mitte Februar von Kosmas Walter in Kehl a. Rh. verkauftes Erzeugnis (der Eimer mit 20 Pfund 
samt Versandkosten zu $16 \mathrm{Mk}$. $20 \mathrm{Pfg}$.) bestand aus gekochten und gequetschten Teilen der roten Rüben, ohne Zusatz von Zucker. Es handelte sich daher um eine sehr leicht verderbliche Ware. Das Erzeugnis war leicht gefärbt und mit schwachem Himbeeraroma versehen. Von dem stark verrosteten Gefäß herrührend, enthielt die Ware nicht unerhebliche Mengen Eisen (Wassergehalt $88 \%$ ).

\section{h) Kaffee-Ersatzmittel u. dergl.}

„Mäntler's Milch-Kaffee Sternmarke, hergestellt aus entfetteter Gebirgstrockenmilch, feinstem Bohnenkaffee und Zusatz von Kaffee-Essenz und Zucker. Der Inhalt des Pakets reicht für 10-12 Tassen. Paket 60 Pfg. Wilh elm Män tler, Stuttgart", $125 \mathrm{~g}$ (19. I. 17). Die Zusammensetzung des Milchkaffee-Pulvers entsprach ungefähr den Angaben. Der Kaffeegehalt war wenig und in Form von Kaffeeextrakt zugesetzt.

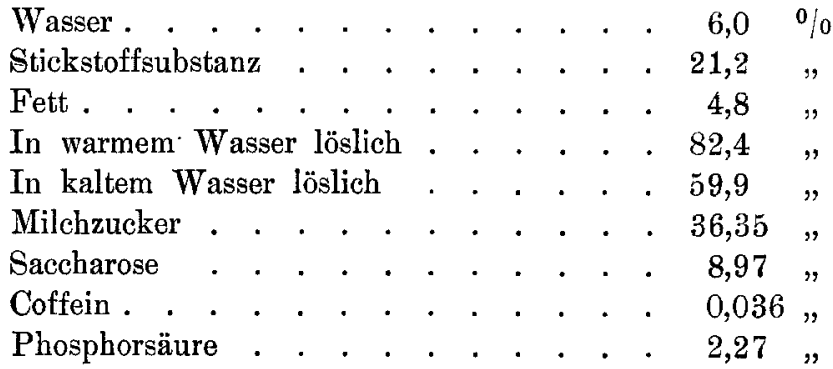

Mikroskopischer Befund: Geringe Mengen von Cichorienteilen erkennbar.

"Weber's Kriegsmischung Kaffee-Ersatz" (14. II. 17), 1 Pfund $=1 \mathrm{Mk} .10 \mathrm{Pfg}$., bestand aus einem Gemenge von Malzkaffee und Cichorie. Es hatte einen sehr hohen Gehalt an Spelzen (Rohfaser 9,1\%).

\section{i) ,Waschmittel's.}

„Burnus, Dr. Röhm's Waschmittel“. „Burnus wäscht Wäsche wunderbar; deutsches Reichspatent. Röhm \& Haas, chemische Fabrik, Darmstadt. Dr. Röhm's Waschmittel Burnus enthält als wirksame Bestandteile Enzyme der Bauchspeicheldrüse, welche die Eigenschaft haben, das Wasser weich zu machen und Eiweiß, Fett, sowie andere Schmutzstoffe auf kaltem Wege zu lösen." $200 \mathrm{~g}=25 \mathrm{Pfg}$. (5. III. 17).

Chemischer Befund:

Qualitativ: Natrium, Calcium, Eisen, Salzsäure, Schwefelsäure und Spuren. von Kohle und Kieselsäure.

Quantitativ:

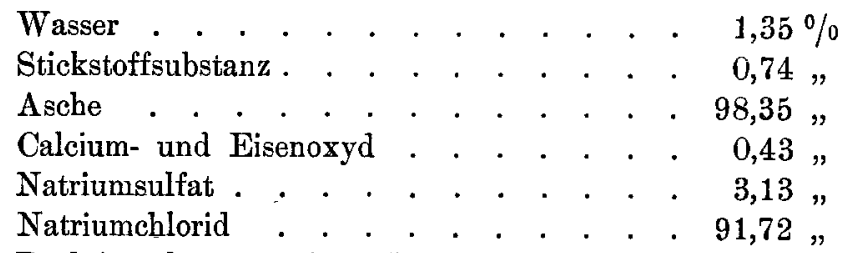

Reaktion der wässerigen Lösung des Salzes: schwach alkalisch.

Das Erzeugnis ist hiernach bis auf etwa $1 \frac{1}{2} \%$ ein mit schwefelsaurem Natrium, etwas Calcium- und Eisencarbonat verunreinigtes Kochsalz. 
„Sauerstoff-Wasch-, Reinigungs- und Bleichmittel Hix“, einer Heilanstalt (Januar 1917) für das Kilo zu $4 \mathrm{Mk}$. angeboten und nach einem beigelegenen Zettel in Stuttgart, Kronenstraße $1 \mathrm{a}$ im Generalvertrieb, bestand zur Haupt. sache aus einem Gemenge von Ätzkali und Pottasche, auf welches geringe Mengen Tetrachlorkohlenstoff aufgetragen waren.

Ein im Oktober 1916 ganz ähnlich angepriesenes Sauerstoff-Waschmittel Hix, verpackt in grünweißen Blechbüchsen zu $1 \mathrm{Mk}$. $20 \mathrm{Pfg}$. mit $10 \mathrm{Päckchen} \mathrm{zu} \mathrm{je}$ 15 g, Fabrikant Fr. Gruner, Seifenfabrik EBlingen, zeigte damals nach unserer Untersuchung eine ganz andere Zusammensetzung; es war ein Gemenge von Soda, Natriumsulfat und Magnesiumsuperoxyd nebst Spuren von Kochsalz und Aluminiumchlorid, auf welche Mineralien kleine Mengen Tetrachlorkohlenstoff aufgetragen waren.

\section{k) „Wäschestärke-Ersatz‘6.}

„Anker-Kochstärke-Ersatz stärkt vorzüglich weiße und farbige Wäsche." Hersteller und Preis nicht genannt. (14. II. 17) $40 \mathrm{~g}=40$ Pfg., bestand aus einer Mischung von Kartoffelmehl mit kleinen Mengen gepulverten Traganths.

„Stärkekraft. Hersteller: Plastische Pläne und Modelle G. m. b. H. Berlin SW 48. Ersatzmittel für Wäschestärke und Glanzștärke" (6. III. 17). Menge: 50 g, Preis 55 Pfg.

Chemischer Befund:

Qualitativ: Calcium in Spuren, Magnesium, Aluminium, Natrium, Koblen. säure, Borsäure, Kieselsäure, Dextrin.

Quantitativ:

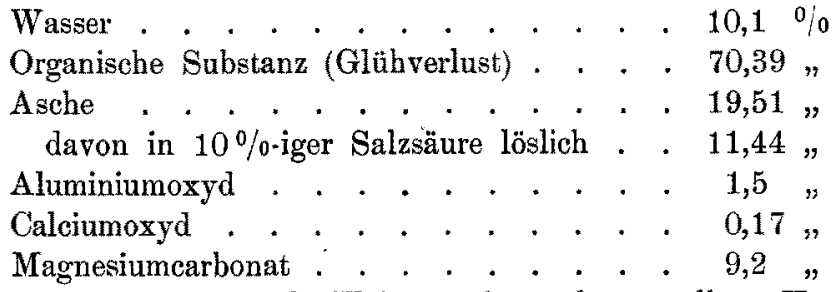

Mikroskopischer Befund: Weizenstärke und verquollene Kartoffelstärke.

Das Erzeugnis war hiernach ein Gemenge von rund $80 \%$ Stärke und Kartoffeldextrin, etwa $9 \%$ Magnesiumsilikat (Talkum), 9\% Magnesiumcarbonat und gegen $2 \%$ Borax. Aluminium und Calcium waren Verunreinigungen. 